Methods Two plasmids were used and compared: pIGneomouse (m)IL-35-GFP (gift from Dario Vignali) and pORFmIL-35, where IL-35 is expressed under CMV or HTLV promoter, respectively. pIGneo-mouse (m)IL-35-GFP contains a 'native' form of IL-35 made of Ebi3 and p35 linked subunits, while pORF-mIL-35contains a 'single chain' construct. In vitro, IL-35 expression was detected in lysate of transfected HEK cells by western blot (p35 or Ebi3) and immunoprecipitation (IP). The in vivo trangene expression was detected by fluorescence analysis of GFP on muscle slides after intra-muscular electrotransfer (ET) of $60 \mu \mathrm{g}$ pIGneo-mIL-35-GFP plasmid. The clinical effect of IL-35 was assessed in collagen-induced arthritis in mice receiving two im ET of $60 \mu \mathrm{g}$ pIGneo-mIL35-GFP or pORF-IL-35, 3 and 18 days after CIA induction. Control groups received the corresponding empty plasmids or PBS. Tregs and Th17 frequencies and phenotypes were performed by flow cytometry in the spleen and lymph nodes (LN) of pORF-IL-35 treated mice at day 34 .

Results Both subunits p35 and Ebi3 were expressed in lysates from either pIGneo-mIL-35-GFP or pORF-IL-35

HEK transfected cells, and formed the IL-35 heterodimer as shown by IP. At least 3 days after intramuscular ET of pIGneo-mIL-35-GFP plasmid, the authors were able to detect GFP expression, revealing in vivo IL-35 expression, on muscle histological slides.

Surprisingly, whatever the plasmid used, IL-35 gene transfer resulted in a statistically significant increase in clinical scores of collagen-induced arthritis as compared to empty plasmid. The underlying cellular mechanisms of this effect were shown to be related to an increased in number and frequency of Th17 cells in the spleen of pORF-mIL-35 treated mice, leading to an increased Th17/Tregs ratio. Interestingly, CD39 expression on Tregs increased (frequency and intensity) in the LN of pORFmIL-35 treated mice as compared to control groups, but CD62L expression also increased (spleen and LN) on these cells.

Conclusion The authors show an unexpected but clear exacerbating effect of IL-35 gene transfer in an autoimmune and inflammatory model of RA. The authors can hypothesise that IL-35, as already known, induced an activation of Tregs by up-regulating CD39 expression on these cells, but that the concomitant up-regulation of CD62L prevent their migration into the inflammatory joint by retaining them in secondary lymphoid organs, thus impeding them from exerting their immunosuppressive role.

\title{
29 IL-35 GENE THERAPY IN A MODEL OF RHEUMATOID ARTHRITIS
}

A Thiolat, ${ }^{1}$ M C Boissier, ${ }^{1,3}$ M Petit, ${ }^{1}$ D Lemeiter, ${ }^{1}$ D Lutomski, ${ }^{2}$ A Denys, ${ }^{1}$ N Bessis'1 'EA4222, Li2P, Paris 13 University, 74 rue Marcel Cachin, Bobigny, France; ${ }^{2}$ UMR 7244, Paris 13 University, 74 rue Marcel Cachin, Bobigny, France, ${ }^{3}$ Rheumatology Department, Avicenne Hospital, Assistance Publique-Hôpitaux de Paris (AP-HP), 125 rue de Stalingrad, Bobigny, France

10.1136/annrheumdis-2011-201230.29

Background and objectives Interleukin (IL)-35 is a novel interleukin described as an immunosuppressive cytokine specifically produced by CD4+FoxP3+ regulatory $\mathrm{T}$ (Tregs) cells but not by conventional $T$ cells. Since Tregs play a major role in autoimmunity maintenance and protects from inflammation, the authors aimed at evaluating the role of IL-35 in a model of rheumatoid arthritis in mice using a non viral gene transfer strategy. 